\title{
Low-dose paclitaxel modulates the cross talk between the JNK and Smad signaling in primary biliary fibroblasts
}

\author{
Jiamei Lu ${ }^{1} \odot$, Liang Yu² $\odot$, Jianhua Shi²* ${ }^{2}$
}

\section{SUMMARY}

OBJECTIVE: The objective of this study was to explore the molecular mechanism underlying the occurrence of benign bile duct stricture and the target of low-dose paclitaxel in the prevention of benign bile duct stricture.

METHODS: Under the stimulation of transforming growth factor beta 1, the expression of collagen type I and connective tissue growth factor were detected on isolated primary fibroblasts. The phosphorylation levels of JNK and Smad2L were detected using Western blot. The effect of low-dose paclitaxel on the transforming growth factor beta 1-induced inhibition of type I collagen and connective tissue growth factor expression and JNK and Smad2L phosphorylation was also observed.

RESULTS: Transforming growth factor beta 1 induced the secretion of type I collagen and connective tissue growth factor as well as JNK phosphorylation in biliary fibroblasts. The JNK inhibitor or siRNA-Smad2 inhibited the transforming growth factor beta 1-induced secretion of type I collagen and connective tissue growth factor. Low-dose paclitaxel inhibited the expression of type I collagen induced by transforming growth factor beta 1 and may inhibit the secretion of collagen in biliary fibroblasts.

CONCLUSION: The activation of JNK/Smad2L induced by transforming growth factor beta 1 is involved in the occurrence of benign bile duct stricture that is mediated by the overexpression of type I collagen and connective tissue growth factor, and low-dose paclitaxel may inhibit the phosphorylation of JNK/Smad2L.

KEYWORDS: Bile duct. Fibroblasts. JNK. Smad. Paclitaxel.

\section{INTRODUCTION}

Anastomotic stricture of the bile duct is one of the main complications after liver transplantation, and the incidence rate is as high as $24.9 \%{ }^{1}$. The incidence of biliary stricture after living-donor liver transplantation in children is as high as $10-35 \%{ }^{2}$. Bile duct stricture severely affects the life and treatment of patients. Therefore, in order to effectively prevent benign bile duct stricture, it is necessary to explore the molecular mechanism underlying the occurrence of benign bile duct stricture.

Recent studies have found that low-dose paclitaxel can prevent tissue fibrosis ${ }^{3-5}$, and clinically, paclitaxel coating has been used to prevent the occurrence of luminal narrowing of blood vessels ${ }^{6}$. Preliminary animal studies of this subject showed that low-dose paclitaxel-coated stents can inhibit the activation of biliary-enteric anastomosis fibroblasts and the secretion of collagen through slow topical administration, but the specific molecular mechanism is still unclear ${ }^{7}$. In this study, we aimed to explore the molecular mechanism underlying the occurrence of benign bile duct stricture. At the same time, the effect of low-dose paclitaxel on the inhibition of type I collagen and connective tissue growth factor (CTGF) secretion investigated in relation to the molecular mechanisms was also obtained.

\section{METHODS}

\section{Isolation, culture, and identification of primary biliary fibroblasts}

The isolation, culture, and identification of primary biliary fibroblasts were performed according to the earlier reported procedure from Wistar rats $^{8}$. The following experiments were performed using the fifth generation cells, and the cells were starved with $10 \mathrm{~mL} / \mathrm{L}$ fetal calf serum for $12 \mathrm{~h}$ before any interventions. SP600125 (Calbiochem, USA) inhibits JNK phosphorylation. Paclitaxel was purchased from Sigma, and transforming growth factor beta 1 (TGF- $\beta 1$ ) was purchased from Peprotech. The experiment was approved by the Animal Ethical Board for Biomedical Experiments of The First Affiliated Hospital of Xi'an Jiaotong University.

\footnotetext{
${ }^{1}$ The Second Affiliated Hospital of Xi'an Jiaotong University, Department of Nephrology - Xi'an, China.

${ }^{2}$ The First Affiliated Hospital of Xi'an Jiaotong University, Department of Hepatobiliary Surgery - Xi'an, China.

*Corresponding author: shi_jian_1981@sina.com

Conflicts of interest: the authors declare there is no conflicts of interest. Funding: The study was supported by the National Natural Science Foundation of China and the Natural Science Foundation of Shanxi Province for funding MRTF-A mediates the epigenetic mechanism of renal tubular epithelial cell transdifferentiation induced by high glucose (81700605) and design and optimization of the surface treatment of a new type of biliary degradable stent (2021JM-269), respectively.

Received on October 08, 2021. Accepted on October 09, 2021.
} 


\section{siRNA transient transfection of siRNA into cells}

Transfection was carried out using Lipofectamine 2000 in accordance with Invitrogen's manufacturer protocol. Western blotting was performed for $48 \mathrm{~h}$ after transfection to determine the inhibitory effect on protein expression.

\section{Enzyme-linked immunosorbent assay test}

Following centrifugation at $1,000 \mathrm{r} / \mathrm{min}$ for $5 \mathrm{~min}$ at $4^{\circ} \mathrm{C}$, the supernatant of medium was collected and stored in a refrigerator at $-20^{\circ} \mathrm{C}$. The type I collagen enzyme-linked immunosorbent assay detection kit (MyBioSource, San Diego, CA, USA) was used to detect the corresponding protein expression level in accordance with the manufacturer's instructions.

\section{Western blotting for the detection of protein levels}

The proteins were separated using SDS-polyacrylamide gel electrophoresis and transferred to a PVDF membrane. The membrane was blocked with $0.1 \%$ BSA in PBS for $1 \mathrm{~h}$ at room temperature and then incubated with the primary antibodies in PBST overnight at $4^{\circ} \mathrm{C}$. The membrane was incubated with a goat anti-rabbit secondary antibody $(1: 2,000)$ for $1 \mathrm{~h}$ at room temperature. Scion NIH image analysis software was used to detect the intensity of the protein bands on Western blot in order to determine the expression level of the target protein.

\section{Statistical analysis}

The experimental results are expressed as mean \pm standard deviation. One-way analysis of variance was used to compare multiple groups of data, while the comparison between the two groups was performed by LSD test using SPSS version 17.0 software. $\mathrm{p}<0.05$ was regarded as statistically significant, and $\mathrm{p}<0.01$ was regarded as the difference of high statistical significance.

\section{RESULTS}

\section{Transforming growth factor beta 1 induces collagen secretion in biliary fibroblasts}

According to Table 1 and Figure 1A, TGF- $\beta 1$ induced the secretion of type I collagen in biliary fibroblasts in a dose-dependent manner, and $10 \mathrm{ng} / \mathrm{mL}$ TGF- $\beta 1$ increased the level of type I collagen secreted by 2.07 times compared with the control group $(\mathrm{p}<0.01)$.

Transforming growth factor beta 1 induces the expression of connective tissue growth factor in biliary fibroblasts in a dose-dependent manner As shown in Figure 1B, TGF- $\beta 1$ induced the CTGF expression in a dose-dependent manner, and we found that $10 \mathrm{ng} / \mathrm{mL}$ TGF- $\beta 1$ increased the CTGF expression by 3.28 times as compared to the control group $(\mathrm{p}<0.01)$.

Transforming growth factor beta 1 induces phosphorylation of JNK in biliary fibroblasts in a dose-dependent manner

As shown in Figure 1C, 3 ng/mL TGF- $\beta 1$ increased the phosphorylation level of JNK as compared to the control group $(\mathrm{p}<0.05)$, whereas $5 \mathrm{ng} / \mathrm{mL}$ TGF- $\beta 1$ increased the phosphorylation level of JNK by 3.85 times compared with the control group $(\mathrm{p}<0.01)$.

Transforming growth factor beta 1-induced phosphorylation of JNK mediates the activation of Smad2L

In comparison with the control group, the phosphorylation of Smad2L was induced in the TGF- $\beta 1$ group $(\mathrm{p}<0.05)$. In contrast, compared with the TGF- $\beta 1$ group, the JNK inhibitor SP600125 inhibited the phosphorylation of Smad2L mediated by TGF- $\beta 1(\mathrm{p}<0.05$; Figure $1 \mathrm{D})$.

\section{Selection of Smad2 gene-specific siRNA for biliary fibroblasts via Smad2 siRNA interference screening}

As shown in Figure 1E, the Western blot following $48 \mathrm{~h}$ post-transfection indicates that Smad2 siRNA-3 had a significant inhibitory effect on the negative control group and the blank control group $(\mathrm{p}<0.01)$, while Smad2 siRNA-1 and -2 had no obvious inhibitory effect. Therefore, we chose Smad2 siRNA 3 for follow-up experiments.

Table 1. Transforming growth factor beta 1 induces collagen secretion in biliary fibroblasts.

\begin{tabular}{|c|c|c|c|c|}
\hline $\begin{array}{l}\text { Concentration of Transforming growth factor } \\
\text { beta } 1(\mathrm{ng} / \mathrm{mL})\end{array}$ & 0 & 1 & 3 & 10 \\
\hline Concentration of collagen (ng/mL) & $2.37 \pm 0.76$ & $2.51 \pm 0.50$ & $3.35 \pm 1.05$ & $4.90 \pm 1.06^{*}$ \\
\hline
\end{tabular}

*The difference is statistically significant $(p<0.01)$ in comparison with the control group. 


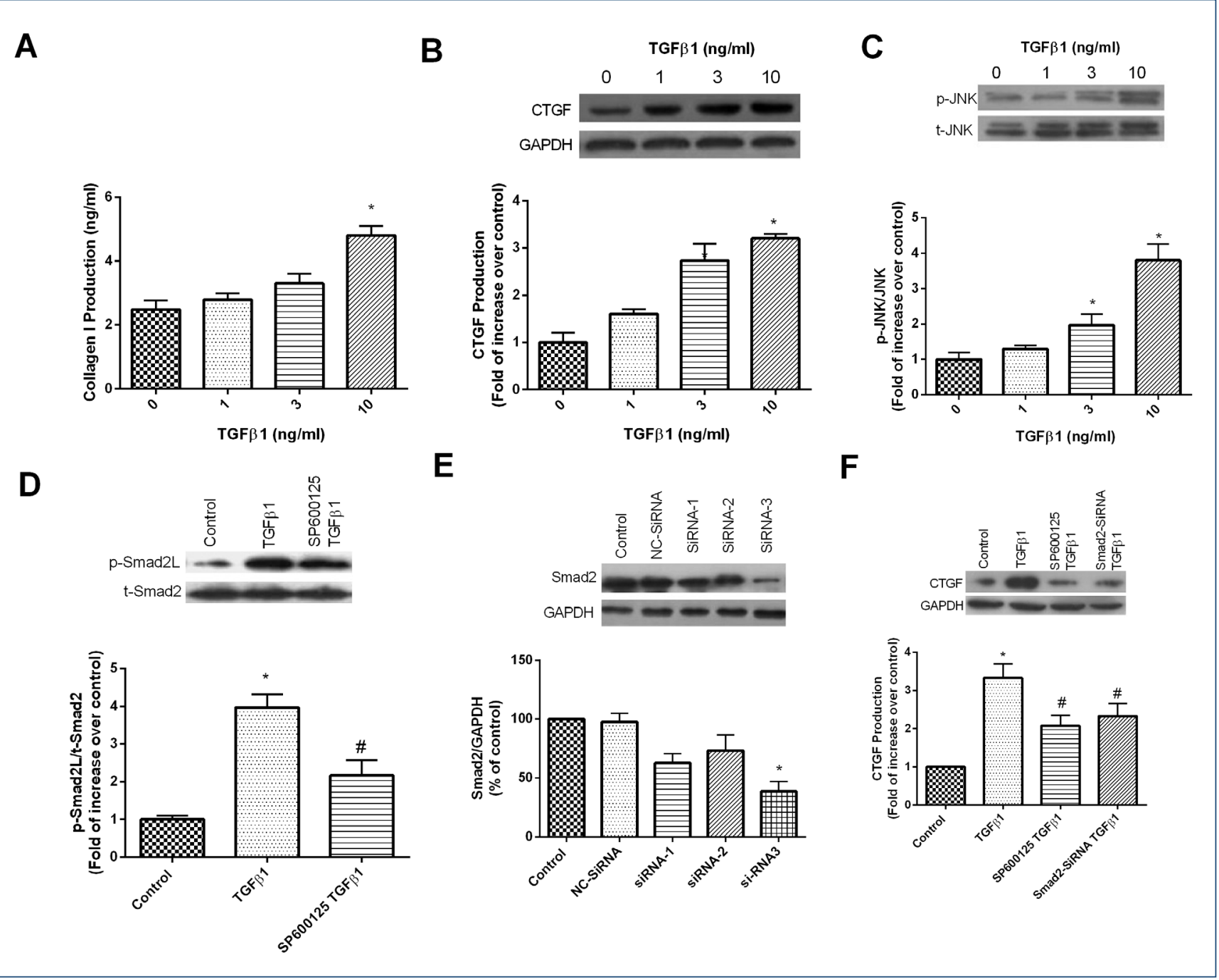

Figure 1. Transforming growth factor comparison: (A) collagen production in biliary fibroblasts, (B) connective tissue growth factor expression in biliary fibroblasts, (C) phosphorylation of JNK in biliary fibroblasts, (D) phosphorylation of JNK mediates the phosphorylation of Smad2L, (E) inhibitory effect of three siRNAs on Smad2 protein, and (F) JNK/Smad2L signaling pathway mediates the secretion of connective tissue growth factor. ${ }^{*}$ The difference is statistically significant $(p<0.01)$ in comparison with the control group. ${ }^{* T h e ~ d i f f e r e n c e ~ i s ~ s t a t i s t i c a l l y ~ s i g n i f i c a n t ~}(p<0.05)$ in comparison with the transforming growth factor beta 1 group.

\section{Transforming growth factor beta 1 activates the JNK/Smad2L signaling pathway and mediates the secretion of connective tissue growth factor}

Compared with the control group, the expression level of CTGF in the TGF- $\beta 1$ group was enhanced $(\mathrm{p}<0.01)$. The JNK inhibitor SP600125 inhibited the expression level of CTGF that was mediated by TGF- $\beta 1$ as compared with the TGF- $\beta 1$ group. In the Smad 2 siRNA group, TGF- $\beta 1$-mediated CTGF expression was also inhibited. Compared with the JNK inhibitor group and the Smad2 siRNA group, no significant difference in the expression level of CTGF mediated by TGF- $\beta 1$ was observed. Thus, it might be suggested that TGF- $\beta 1$ mediates the expression of CTGF by activating the JNK/Smad2L signaling pathway (Figure 1F).

\section{Transforming growth factor beta 1 induces} the phosphorylation of JNK and Smad2L and mediates the expression of type I collagen

Figure $2 \mathrm{~A}$ shows that compared with the control group, the expression level of type I collagen of the TGF- $\beta 1$ group was increased $(\mathrm{p}<0.01)$. The JNK inhibitor SP600125 inhibited the expression level of type I collagen mediated by TGF- $\beta 1$. In the Smad2 siRNA group, the expression level of type I collagen mediated by TGF- $\beta 1$ was also inhibited. Therefore, these 


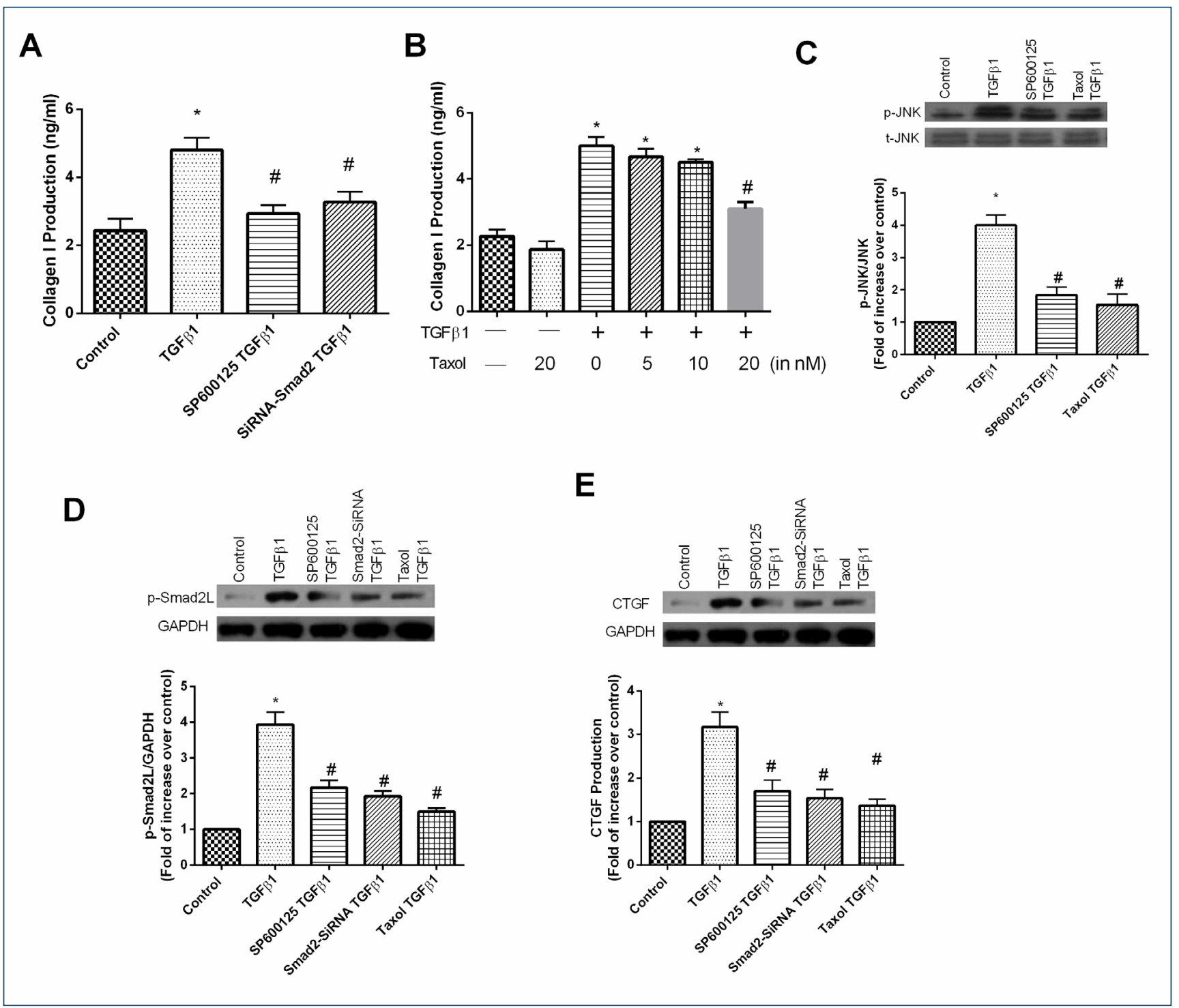

Figure 2. Molecular mechanism explained that (A) transforming growth factor beta 1 induces the phosphorylation of JNK and Smad2L and mediates the secretion of type I collagen, (B) paclitaxel inhibits the expression of type I collagen induced by transforming growth factor beta 1, (C) JNK phosphorylation induced by transforming growth factor beta 1, (D) transforming growth factor beta-mediated phosphorylation of Smad2L, and (E) transforming growth factor beta 1-induced connective tissue growth factor expression. ${ }^{*}$ The difference is statistically significant $(p<0.01)$ in comparison with the control group; \#The difference is statistically significant $(p<0.05)$ in comparison with the transforming growth factor beta 1 group.

findings suggest that TGF- $\beta 1$ mediates the expression of type I collagen by activating the JNK/Smad2L signaling pathway.

\section{Paclitaxel inhibits transforming growth factor beta 1-mediated secretion of type I collagen in biliary fibroblasts in a dose-dependent manner}

As shown in Figure 2B, $10 \mathrm{ng} / \mathrm{mL}$ TGF- $\beta 1$ significantly increased the secretion of type I collagen in biliary fibroblasts as compared with the control group. Paclitaxel dose-dependently inhibited the TGF- $\beta 1$-mediated secretion of type I collagen in biliary fibroblasts. Particularly, $20 \mathrm{nmol} / \mathrm{L}$ paclitaxel significantly reduced the secretion of type I collagen in biliary fibroblasts compared with the TGF- $\beta 1$ group, suggesting that $20 \mathrm{nmol} / \mathrm{L}$ paclitaxel has a significant inhibitory effect on TGF- $\beta 1$-induced type I collagen secretion in biliary fibroblasts. In the absence of TGF- $\beta 1$ stimulation, paclitaxel has no significant effect on type I collagen of biliary fibroblasts (compared with the blank control group, $\mathrm{p}>0.05$ ). Therefore, $20 \mathrm{nmol} / \mathrm{L}$ paclitaxel was chosen for follow-up experimental research. 


\section{Effect of paclitaxel on JNK phosphorylation} induced by transforming growth factor beta 1

The results in Figure $2 \mathrm{C}$ indicate that paclitaxel can significantly inhibit the level of JNK phosphorylation induced by TGF- $\beta 1$ ( $p<0.05$, compared with the TGF- $\beta 1$ group). However, its effect was not statistically different compared with the JNK inhibitor group $(\mathrm{p}>0.05)$.

\section{Effect of paclitaxel on transforming growth factor beta 1-mediated phosphorylation of Smad2L}

In order to explore whether paclitaxel can inhibit the phosphorylation of Smad2L induced by TGF- $\beta 1$, the results in Figure $2 \mathrm{D}$ indicate that paclitaxel can significantly inhibit the phosphorylation of Smad2L induced by TGF- $\beta 1(\mathrm{p}<0.05$, compared with the TGF- $\beta 1$ group). Nevertheless, there is no statistical difference in its effect when compared with the JNK inhibitor group or siRNA group ( $\mathrm{p}>0.05)$.

\section{Paclitaxel inhibits the transforming growth factor beta 1-mediated expression of connective tissue growth factor in biliary fibroblasts}

To examine whether paclitaxel can inhibit TGF- $\beta 1$-induced expression of CTGF, the results in Figure 2E indicate that paclitaxel can significantly inhibit the TGF- $\beta 1$-induced secretion of CTGF compared with the TGF- $\beta 1$ group, and its effect is similar to that in the TGF- $\beta 1$ group. Nevertheless, there was no statistical difference when compared with the JNK inhibitor group or the siRNA group ( $>0.05$ ), indicating that paclitaxel inhibits CTGF secretion possibly via the suppression of the phosphorylation of JNK and Smad2L.

\section{DISCUSSION}

A growing body of evidence suggests that non-Smad pathways are involved in the transduction of TGF- $\beta$ signals. These nonSmad pathways can be directly mediated by TGF- $\beta$ or can involve in the regulation of TGF- $\beta /$ Smad signal transduction, thereby bestowing TGF- $\beta$ with multiple biological functions ${ }^{9}$. Recently, Lessard et al. found that JNK can adjust skeletal muscle remodeling through Smad $2^{10}$. Shapira et al. reported that increased phosphorylation of JNK can increase the translation and activation of $S m a d 2 / 3$ induced by TGF- $\beta^{11}$. Smads protein is composed of conserved MH1, MH2, and intermediate linking regions and a central mediator of TGF- $\beta$ signal transduction. TGF- $\beta$ type I receptor (T $\beta \mathrm{RI})$ phosphorylates the $\mathrm{COOH}$-terminal serine residues of Smads protein activated by membrane receptors, and the intermediate linking region can be phosphorylated by Ras-related kinases, such as ERK, JNK, P38, and CDK4 $4^{12,13}$.
TGF- $\beta 1$ not only directly mediates the phosphorylation of the $\mathrm{COOH}$-terminal serine residues of Smads by activating the T $\beta \mathrm{RI}$ but also promotes the activation of Ras-related kinases through TGF- $\beta$ activated kinase 1 and mediates the phosphorylation of Smads protein linking region to coordinate TGF- $\beta 1$ signal transduction ${ }^{13}$. The TGF- $\beta 1$-mediated Ras-related kinase-dependent Smad2/3 intermediate linking region promotes not only cell proliferation but also tissue fibrosis. Abe et al. found that ERK1/2 contributed to the increase of the collagen synthesis mediated by TGF- $\beta 1$ in cardiac fibroblasts, which was accompanied by phosphorylation of the intermediate linking region of Smad2, while the loss of ERK significantly down-regulated the expression of collagen ${ }^{14}$. In this study, TGF- $\beta 1$ induced the expression of CTGF and type I collagen in a dose-dependent manner, accompanied by increased phosphorylation of JNK and Smad2L, and inhibition of phosphorylation of JNK or Smad2L could reduce the expression of CTGF and type I collagen. JNK inhibitors can also inhibit the phosphorylation of Smad2L induced by TGF$\beta 1$, suggesting that the TGF- $\beta 1$-induced expression of CTGF and type I collagen in biliary fibroblasts is mediated by the JNK/ Smad2L pathway. Hence, TGF- $\beta 1 / \mathrm{JNK} / \mathrm{Smad} 2 \mathrm{~L}$ is involved in the occurrence of benign bile duct stricture.

Recent studies suggest that low-dose paclitaxel (10-50 nM) is able to prevent tissue fibrosis by regulating the TGF- $\beta 1 / \mathrm{Smads}$ signaling pathway and inhibiting the phosphorylation of $S \operatorname{mad} 2 / 3$ to reduce the deposition of collagen ${ }^{15-17}$. The results of this study suggest that low-dose paclitaxel inhibits the TGF- $\beta 1$-induced secretion of collagen in a dose-dependent manner, accompanied by the inhibition on phosphorylation of JNK and Smad2L. Therefore, we found that low-dose paclitaxel-induced inhibitory effect on the secretion of collagen may be achieved by inhibiting the phosphorylation of JNK and Smad2L, and low-dose paclitaxel may regulate the TGF- $\beta 1 / S$ mads signaling pathway through JNK to downregulate collagen secretion in human biliary fibroblasts.

\section{CONCLUSION}

Our present study demonstrated that activation of JNK/Smad2L induced by TGF- $\beta 1$ is involved in the occurrence of benign bile duct stricture that is mediated by the overexpression of type I collagen and CTGF, and low-dose paclitaxel may inhibit the phosphorylation of JNK/Smad2L.

\section{AUTHORS' CONTRIBUTIONS}

JL, LY: Performed the study, Analyzed the data, Wrote the initial draft of this manuscript. JS: Conceptualized and supervised the study, Approved the final version of this manuscript. 


\section{REFERENCES}

1. Jarlot-Gas C, Muscari F, Mokrane FZ, Del Bello A, Culetto A, Buscail E, et al. Management of anastomotic biliary stricture after liver transplantation and impact on survival. HPB (Oxford). 2021;23(8):1259-68. https://doi.org/10.1016/j.hpb.2020.12.008

2. Yoshizumi T, Harada N, Mori M. Biliary stricture: the achilles heel of pediatric living donor liver transplantation. Transplantation. 2019;103(9):1758-9. https://doi.org/10.1097/ TP.0000000000002573

3. Wang M, Chen L, Huang W, Jin M, Wang Q, Gao Z, et al. Improving the anti-keloid outcomes through liposomes loading paclitaxelcholesterol complexes. Int J Nanomed. 2019;14:1385-400. https:// doi.org/10.2147/IJN.S195375

4. Luan J, Zhang Z, Shen W, Chen Y, Yang X, Chen X, et al. Thermogel loaded with low-dose paclitaxel as a facile coating to alleviate periprosthetic fibrous capsule formation. ACSAppl Mater Interfaces. 2018;10(36):30235-46. https://doi.org/10.1021/acsami.8b13548

5. Sharawy MH, El-Awady MS, Makled MN. Protective effects of paclitaxel on thioacetamide-induced liver fibrosis in a rat model. J Biochem Mol Toxicol. 2021;35(5):e22745. https://doi.org/10.1002/ jbt.22745

6. Krishnan P, Purushothaman KR, Purushothaman M, Tarricone A, Chen S, Singla S, et al. Histological features of restenosis associated with paclitaxel drug-coated balloon: implications for therapy. Cardiovasc Pathol. 2019;43:107139. https://doi.org/10.1016/j. carpath.2019.06.003

7. Shi J, Lv Y, Yu L, Zhang B, Zhang X, Fan C, et al. Interest of a new biodegradable stent coated with paclitaxel on anastomotic wound healing after biliary reconstruction. Eur J Gastroenterol Hepatol. 2013;25(12):1415-23. https://doi.org/10.1097/ MEG.0b013e328361eb51

8. Sirica AE. Bile duct epithelial cell culture. Methods Mol Biol. 2002;188:37-52. https://doi.org/10.1385/1-59259-185-x:37
9. $\mathrm{Yu}$ Y, Feng $\mathrm{XH}$. TGF- $\beta$ signaling in cell fate control and cancer. Curr Opin Cell Biol. 2019;61:56-63. https://doi.org/10.1016/j. ceb.2019.07.007

10. Lessard SJ, MacDonald TL, Pathak P, Han MS, Coffey VG, Edge J, et al. JNK regulates muscle remodeling via myostatin/SMAD inhibition. Nat Commun. 2018;9(1):3030. https://doi.org/10.1038/ s41467-018-05439-3

11. Shapira KE, Ehrlich M, Henis YI. Cholesterol depletion enhances TGF- $\beta$ Smad signaling by increasing c-Jun expression through a PKR-dependent mechanism. Mol Biol Cell. 2018;29(20):2494-507. https://doi.org/10.1091/mbc.E18-03-0175

12. Matsuzaki K. Smad phosphoisoform signals in acute and chronic liver injury: similarities and differences between epithelial and mesenchymal cells. Cell Tissue Res. 2012;347(1):225-43. https:// doi.org/10.1007/s00441-011-1178-6

13. Yoshida K, Matsuzaki K, Murata M, Yamaguchi T, Suwa K, Okazaki K. Clinico-pathological importance of TGF- $\beta$ /phospho-smad signaling during human hepatic fibrocarcinogenesis. Cancers (Basel). 2018;10(6):183. https://doi.org/10.3390/cancers10060183

14. Abe H, Takeda N, Isagawa T, Semba H, Nishimura S, Morioka MS, et al. Macrophage hypoxia signaling regulates cardiac fibrosis via Oncostatin M. Nat Commun. 2019;10(1):2824. https://doi. org/10.1038/s41467-019-10859-w

15. Mojsilovic S, Tosic M, Mojsilovic S, Zivanovic M, Bjelica S, Srdic Rajic T, et al. Paclitaxel inhibits transforming growth factor- $\beta$ increased urokinase-type plasminogen activator expression through p38 MAPK and RAW 264.7 macrophage migration. J BUON. 2020;25(2):1257-65. PMID: 32521934

16. Son SS, Kang JS, Lee EY. Paclitaxel ameliorates palmitateinduced injury in mouse podocytes. Med Sci Monit Basic Res. 2020;26:e928265. https://doi.org/10.12659/MSMBR.928265

17. Lyu SC, Wang J, Zhou L, Zhu JQ, Pan F, Jiang T, et al. Mechanism of scar formation following Roux-en-Y choledochojejunostomy in a novel rat model of obstructive jaundice. Ann Transl Med. 2021;9(6):456. https://doi.org/10.21037/atm-20-5135 